Article

\title{
Approximate Solutions and Symmetry of a Two-Component Nonlocal Reaction-Diffusion Population Model of the Fisher-KPP Type
}

\author{
Alexander V. Shapovalov ${ }^{1,2, *,+}$ (D) and Andrey Yu. Trifonov ${ }^{3,+}$ \\ 1 Department of Theoretical Physics, Tomsk State University, 1 Novosobornaya Sq., 634050 Tomsk, Russia \\ 2 Department of Physics and Mathematics, Tomsk State Pedagogical University, 60 Kievskaya St., \\ 634041 Tomsk, Russia \\ 3 Department of Mathematics and Informatics, Tomsk Polytechnic University, 30 Lenin ave., \\ 634034 Tomsk, Russia; atrifonov@tpu.ru \\ * Correspondence: shpv@phys.tsu.ru; Tel.: +7-382-252-1754 \\ + These authors contributed equally to this work.
}

Received: 17 February 2019; Accepted: 8 March 2019; Published: 12 March 2019

check for updates

\begin{abstract}
We propose an approximate analytical approach to a $(1+1)$ dimensional two-component system consisting of a nonlocal generalization of the well-known Fisher-Kolmogorov-PetrovskiiPiskunov (KPP) population equation and a diffusion equation for the density of the active substance solution surrounding the population. Both equations of the system have terms that describe the interaction effects between the population and the active substance. The first order perturbation theory is applied to the system assuming that the interaction parameter is small. The Wentzel-Kramers-Brillouin (WKB)-Maslov semiclassical approximation is applied to the generalized nonlocal Fisher-KPP equation with the diffusion parameter assumed to be small, which corresponds to population dynamics under certain conditions. In the framework of the approach proposed, we consider symmetry operators which can be used to construct families of special approximate solutions to the system of model equations, and the procedure for constructing the solutions is illustrated by an example. The approximate solutions are discussed in the context of the released activity effect variously debated in the literature.
\end{abstract}

Keywords: nonlocal Fisher-KPP model; reaction-diffusion; semiclassical approximation; perturbation method; symmetries; released activity

\section{Introduction}

Investigations of non-local models of reaction-diffusion (RD) systems with long-range interactions are a developing trend in modern nonlinear physics and mathematics ranging from condensed matter physics to physics of living systems.

RD systems are in the focus of researchers due to the spatial and temporal patterns that can be formed in such systems in the process of their evolution under certain conditions. The pattern formation in RD systems is considered as an example of self-organisation in nonlinear systems and, in particular, in biological systems [1].

A relatively simple example of RD systems is the dynamics of microbiological populations (bacteria, viruses, or cells). Many models of such RD systems are built on the basis of the well-known classical Fisher-Kolmogorov-Petrovskii-Piskounov (Fisher-KPP) equation describing space-time evolution of the population density [2,3]. In the classical Fisher-KPP model, the population dynamics of a single species with a local interaction between individuals is considered. 
In some cases, the dynamics of non-local RD systems is modeled by integro-differential equations (IDEs) with partial derivatives and variable coefficients. Non-local generalizations of the classical Fisher-KPP equation were used in the study of cell population dynamics, in particular, in cancer modeling (see, e.g., [4-8] and references therein).

In view of the mathematical complexity of such equations, there is great interest in developing analytical methods for obtaining solutions for RD systems, especially, with long-range interactions.

The set of known analytical methods and approaches for RD models with both local and especially with non-local interactions is extremely limited and applies as a rule only to very simplified cases. An example of an exact analytical approach is the Lie group analysis of partial differential equations (PDEs) [9-12] that allowed the authors to find invariance groups and their corresponding particular solutions to PDEs.

Extending the scope of the Lie-group methods to integro-differential equations by invoking various means of bringing IDEs to PDEs (see, e.g., [13-17]) made it possible to apply the group and symmetry analysis to non-local RD models with long-range interactions, including non-local generalizations of the Fisher-KPP equation [18].

Approximate methods and approaches are also promising for the development of analytical methods to study complex and especially non-local RD models. We will focus on the Wentzel-KramersBrillouin (WKB)-Maslov method of semiclassical asymptotics [19-21], which has been effectively applied to the non-local generalization of the Fisher-KPP equation (see $[6,7,18,22,23]$ and references therein):

$$
-u_{t}(x, t)+D u_{x x}(x, t)+a(x, t) u(x, t)-\varkappa u(x, t) \int_{-\infty}^{\infty} b(x, y) u(y, t) d y=0 .
$$

For simplicity we restrict ourself to the (1+1)-dimensional case. In (1), $x$ and $t$ are the space and the time variable, respectively; the real function $u(x, t)$ denotes population density; $a(x, t)$ and $b(x, y, t)$ are given as infinitely smooth functions increasing, as $|x|,|y| \rightarrow \infty$, no faster than the polynomial; $\varkappa(>0)$ is a real nonlinearity parameter; $D$ is the diffusion coefficient; $u_{t}=\partial u / \partial t, u_{x}=\partial u / \partial x$, $u_{x x}=\partial^{2} u / \partial x^{2}$. The term $-\varkappa u(x, t) \int_{-\infty}^{\infty} b(x, y) u(y, t) d y$ describes the non-local competition losses in the population and is characterized by the influence function $b(x, y)$; the function $u(x, t)$, together with all its derivatives, is assumed to decrease, faster than any negative power of $|x|$, as $|x|$ tends to infinity.

Approximate semiclassical solutions were constructed for the one-dimensional $[22,23]$ and multidimensional $[6,7]$ non-local Fisher-KPP equations. In studies of the dynamics of biological populations, it is important to investigate how external factors can influence population growth.

In [24] a model was proposed in which the population growth occurs in a solution of an active substance surrounding the population and interacting with it. The dynamics of the active substance density is described by the diffusion equation supplemented by a corresponding term to describe the interaction between the substance and population densities. A similar term is included in the non-local Fisher-KPP equation. The system of model equations was solved numerically.

The proposed model may have interesting applications in a theory of population dynamics when the self-consistent external control factors of the active substance of different nature impact the dynamics. One of the important applications of the model is to analyze the effects of external factors on cell populations in the context of cancer research $[4,5]$. The model can also contribute to studying the characteristics of the effect of low and ultra-low concentrations of an active substance on cell population dynamics due to the phenomenon of released activity discussed in the literature (e.g., [25-27]).

In this study we offer an approximate analytical approach to the model proposed in [24]. A two-parameter approximation is developed for the system of model equations. The approach uses the perturbation method with a small parameter to describe the interaction between population 
density and active substance density and the WKB-Maslov method of semiclassical asymptotics assuming a weak diffusion for solving the non-local Fisher-KPP type equation with an interaction term [23]. Note that the assumption of weak diffusion corresponds to population dynamics under certain real conditions (see, e.g., [1]).

In the framework of the formalism of semiclassical asymptotics, we discuss the applicability of the method for constructing symmetry operators developed in $[28,29]$ to the system of model equations under consideration to extend a set of approximate analytical solutions.

To summarize, we would like to note that the approximate analytical approach offered here for $\mathrm{RD}$ population dynamical systems with long-range interactions can have wider applications in solving various problems of nonlinear physics ranging from quantum matter wave models to cosmology (see, e.g., $[30,31]$ and references therein).

The paper is arranged as follows. In Section 2, basic definitions and the model equations describing the time evolution of the population density and the active substance density are introduced. Expressions are derived for the leading terms and the first-order corrections of the perturbation power series in a small interaction parameter for the Cauchy problem. In Section 3, the WKB-Maslov semiclassical approximation method with the diffusion parameter assumed to be small is applied to the generalized nonlocal Fisher-KPP equation describing the leading-order term in the perturbed solution for the Cauchy problem. The symmetry operators of the semiclassically reduced nonlocal Fisher-KPP equation are considered in Section 4. The calculations of the first-order perturbation solution are illustrated by an example discussed in Section 5. Finally, concluding remarks are given in Section 6.

\section{Model Equations and Perturbation Theory}

Consider the two-component one-dimensional non-local RD-type model describing the dynamics of a population of density $u(x, t)$ interacting with an active substance of density $v(x, t)$ surrounding the population.

The population dynamics are governed by the non-local generalized equation of the Fisher-KPP type (1) with an interaction term, and the active substance dynamics are described by the diffusion equation with an interaction term.

To write the system of model equations, we introduce the nonlinear operator $\hat{F}(D)$ of the Fisher-KPP Equation (1) as

$$
\hat{F}(D) u(x, t)=\hat{M}(D) u(x, t)+a(x, t) u(x, t)-\varkappa u(x, t) \int_{-\infty}^{\infty} b(x, y) u(y, t) d y .
$$

Here $\hat{M}(D)$, given by

$$
\hat{M}(D)=-\partial_{t}+D \partial_{x x}
$$

is the operator of the well-known linear diffusion equation with the diffusion coefficient $D ; \partial_{t}=\partial / \partial t$ and $\partial_{x x}=\partial^{2} / \partial x^{2}$.

In terms of operators (2) and (3), the system of model equations describing the time evolution of the population density $u(x, t)$ and active substance density $v(x, t)$ can be written in dimensionless form as [24]

$$
\begin{array}{r}
\hat{F}\left(D_{1}\right) u(x, t)-\lambda f(u, v)(x, t)=0, \\
\hat{M}\left(D_{2}\right) v(x, t)-\lambda g(u, v)(x, t)=0 .
\end{array}
$$

Here, $D_{1}$ and $D_{2}$ are the diffusion coefficients for the population and the active substance, respectively; $f(u, v)(x, t)$ and $g(u, v)(x, t)$ are considered as binary operators that model the interaction 
of the fields $u(x, t)$ and $v(x, t)$ in Equations (4) and (5). The minus sign of $\lambda f$ in (4) implies that the action of $v$ on $u$ suppresses the growth of the population, and the term $-\lambda g$ in (5) indicates that the consumption of $v$ by the population $u$ lowers the density $v$. The specific form of $f$ and $g$ may vary depending on the specific problem. For example, the interaction terms

$$
\begin{aligned}
& f(u, v)(x, t)=\mu u(x, t) \int_{0}^{t} u(x, \tau) v(x, \tau) d \tau, \\
& g(u, v)(x, t)=v u(x, t) v(x, t)
\end{aligned}
$$

were used in [24] in modelling the dynamics of a cellular population whose growth is suppressed by an active substance in terms of the system (4) and (5). Here $\mu$ and $v$ are dimensional factors.

A simpler model with an interaction similar to (6) was explored in [32]. The form of the interaction term $f(u, v)(x, t)$ in (6) can be considered as an accumulated action of $v$ on $u$ over the time interval $[0, t]$, and expression (7) implies that the population $(u)$ consumes the substance $(v)$ directly at contact. For convenience, the interaction parameter $\lambda$ is introduced explicitly in Equations (4) and (5).

The form of IDEs (4), (5) indicates that the real way to find analytical solutions for this system may be in the use of a suitable approximation. In this regard, let us assume that the interaction parameter $\lambda$ is small and apply the regular perturbation theory to the Cauchy problem for the system (4), (5) with initial data

$$
u(x, 0)=\varphi(x), \quad v(x, 0)=\psi(x) .
$$

An approximate solution to the Cauchy problem (8) is sought as a formal perturbation power series in $\lambda$ :

$$
u(x, t)=\sum_{k=0}^{\infty} \lambda^{k} u^{(k)}(x, t), \quad v(x, t)=\sum_{k=0}^{\infty} \lambda^{k} v^{(k)}(x, t) .
$$

The first few terms of the expansions (9) represent the perturbation solution of the system (4) and (5) or an approximation. The functions $u^{(0)}(x, t)$ and $v^{(0)}(x, t)$ are the leading order terms of the perturbation series. In the first-order perturbation theory, the expansions (9) contain the first two terms with $k=0,1$. We limit our consideration to the first approximation.

Substituting the expansions (9) in the system (4) and (5), collecting terms in order of powers of $\lambda$, and equating them to zero, we obtain the following sets of equations for obtaining an approximate solution in the first-order perturbation theory:

$$
\begin{gathered}
\hat{F}\left(D_{1}\right) u^{(0)}(x, t)=0, \\
\hat{M}\left(D_{2}\right) v^{(0)}(x, t)=0,
\end{gathered}
$$

and

$$
\begin{aligned}
& -u_{t}^{(1)}(x, t)+D_{1} u_{x x}^{(1)}(x, t)+a(x, t) u^{(1)}(x, t)-\varkappa u^{(1)}(x, t) \int_{-\infty}^{\infty} b(x, y) u^{(0)}(y, t) d y \\
& -\varkappa u^{(0)}(x, t) \int_{-\infty}^{\infty} b(x, y) u^{(1)}(y, t) d y-f\left(u^{(0)}, v^{(0)}\right)(x, t)=0 \\
& \hat{M}\left(D_{2}\right) v^{(1)}(x, t)-g\left(u^{(0)}, v^{(0)}\right)(x, t)=0 .
\end{aligned}
$$

Equations (10) and (11) describe the leading-order terms, and Equations (12) and (13) describe the first-order terms of the approximate solution (9) of the Cauchy problem (4), (5), and (9). 
Applying the initial conditions (8) to the perturbation series (9), we obtain

$$
\begin{aligned}
& u^{(0)}(x, 0)=\varphi(x), \\
& v^{(0)}(x, 0)=\psi(x)
\end{aligned}
$$

for the system (10), (11), and for the system (12), (13) we have

$$
\begin{aligned}
& u^{(1)}(x, 0)=0, \\
& v^{(1)}(x, 0)=0 .
\end{aligned}
$$

The solution of the Cauchy problems for the homogeneous diffusion Equation (11) subject to the non-zero initial condition (15) and the non-homogeneous Equation (13) subject to the zero initial condition (17) is standard and causes no problems.

It is nontrivial to find a solution of the Cauchy problem for the non-local nonlinear homogeneous unperturbed Fisher-KPP Equation (10) with the non-zero initial condition (14) and for the linear non-local inhomogeneous Equation (12) for the first-order correction with the zero initial condition (16).

In the next section, we will apply the WKB-Maslov method of semiclassical asymptotics developed in $[22,23]$ under the assumption of weak diffusion in the population dynamics to solving the both Cauchy problems.

\section{Semiclassical Approximation for the Cauchy Problem}

\subsection{Semiclassical Solution of the Non-Local Fisher-KPP Equation}

A semiclassical solution to the Cauchy problem for the non-local Fisher-KPP Equation (10) was constructed in $[22,23]$ in the class of trajectory concentrated functions $\mathcal{P}_{t}^{D_{1}}$ defined by their common element:

$$
\mathcal{P}_{t}^{D_{1}}=\left\{\Phi: \Phi\left(x, t, D_{1}\right)=\varphi\left(\frac{\Delta x}{\sqrt{D}_{1}}, t, D_{1}\right) \exp \left[\frac{1}{D_{1}} S\left(t, D_{1}\right)\right]\right\},
$$

where the following designations are used: $\Delta x=x-X\left(t, D_{1}\right)$, the real function $\varphi\left(\eta, t, D_{1}\right)$ belongs to the Schwarz space $\mathbb{S}$ in a variable $\eta \in \mathbb{R}^{1}$, smoothly depends on $t$, and regularly depends on $\sqrt{D_{1}}$ as $D_{1} \rightarrow 0$. To specify initial functions, we introduce a class $\mathcal{P}_{0}^{D_{1}}$ defined by the relation

$$
\mathcal{P}_{0}^{D_{1}}=\left.\mathcal{P}_{t}^{D_{1}}\right|_{t=0}
$$

Below we briefly consider how to construct the leading term of the semiclassical asymptotic solution to the Cauchy problem (10) and (14) following [23].

The real functions $S\left(t, D_{1}\right)$ and $X\left(t, D_{1}\right)$ characterize the class $\mathcal{P}_{t}^{D_{1}}$ and are to be determined when asymptotic solutions are constructed. These functions are regular in the parameter $\sqrt{D_{1}}$ in a neighborhood of $D_{1}=0$ (each of them can be expressed as a power series in $\sqrt{D_{1}}$ ).

All functions of the class $\mathcal{P}_{t}^{D_{1}}$ singularly depend on the asymptotic parameter $D_{1}$ and are concentrated, as $D_{1} \rightarrow 0$, in a neighborhood of a point moving in the coordinate space along a curve given by the Equation $x=X\left(t, D_{1}\right)$. In accordance with this property, we call $\mathcal{P}_{t}^{D_{1}}$ the class of trajectory concentrated functions (TCF) (see [23]).

For the functions of the class $\mathcal{P}_{t}^{D_{1}}$, the following asymptotic estimates hold [23,33,34]:

$$
\hat{p}^{k} \Delta x^{l}=\widehat{O}\left(D_{1}^{(k+l) / 2}\right), \quad \hat{T}\left(X\left(t, D_{1}\right), t\right)=\widehat{O}\left(D_{1}\right),
$$

where

$$
\hat{p}=D_{1} \partial_{x}, \quad \hat{T}\left(X\left(t, D_{1}\right), t\right)=D_{1} \partial_{t}+\dot{X}\left(t, D_{1}\right) D_{1} \partial_{x}-\dot{S}\left(t, D_{1}\right),
$$

and $\widehat{O}\left(D_{1}^{\mu}\right)$ is an operator $\hat{F}$ such that $\frac{\|\hat{f} \phi\|}{\|\phi\|}=O\left(D_{1}^{\mu}\right), \quad \phi \in \mathcal{P}_{t}^{D_{1}}$. 
The norm $\|u\|$ is understood in the sense of the space $L_{2}$.

From (20) it follows that

$$
\hat{p}=\widehat{O}\left(\sqrt{D_{1}}\right), \quad \Delta x=\widehat{O}\left(\sqrt{D_{1}}\right) .
$$

We seek an asymptotic solution to the Cauchy problem (10) and (14) in the class of functions $\mathcal{P}_{t}^{D_{1}}$ as $D_{1} \rightarrow 0$. The initial function $\varphi(x)$ is taken from the class $\mathcal{P}_{0}^{D_{1}}$ of the form (19).

Whenever not confusing, we will occasionally omit the explicit dependence of functions on the parameter $D_{1}$ for brevity.

For the Fisher-KPP Equation (10), we use the formal power series in $\Delta x$ for the functions $a(x, t)$ and $b(x, y)$ entering into the operator $\hat{L}$ of the form (2). In view of that the estimates (20)-(22) are correct in the class of functions $\mathcal{P}_{t}^{D_{1}}$, we have

$$
\begin{aligned}
a(x, t) & =a_{0}(t)+\Delta x a_{1}(t)+\widehat{O}\left(D_{1}\right), \\
b(x, y) & =b_{0}(t)+\Delta x b_{1,0}(t)+\Delta y b_{0,1}(t)+\widehat{O}\left(D_{1}\right) .
\end{aligned}
$$

Here, $a_{0}(t)=a_{0}\left(t, D_{1}\right)=a\left(X\left(t, D_{1}\right), t\right), a_{1}(t)=a_{1}\left(t, D_{1}\right)=\partial a(x, t) /\left.\partial x\right|_{x=X\left(t, D_{1}\right)}, b_{0}(t)=$ $b_{0}\left(t, D_{1}\right)=b\left(X\left(t, D_{1}\right), X\left(t, D_{1}\right)\right), b_{1,0}(t)=b_{1,0}\left(t, D_{1}\right)=\partial b(x, y) /\left.\partial x\right|_{x=y=X\left(t, D_{1}\right)}, b_{0,1}(t)=$ $b_{0,1}\left(t, D_{1}\right)=\partial b(x, y) /\left.\partial y\right|_{x=y=X\left(t, D_{1}\right)}, \Delta y=y-X\left(t, D_{1}\right)$.

Let $\sigma^{(0)}(t)=\sigma^{(0)}\left(t, D_{1}\right), x^{(0)}(t)=x^{(0)}\left(t, D_{1}\right), \alpha^{(k)}(t)=\alpha^{(k)}\left(t, D_{1}\right)$ be the moments of a solution $u^{(0)}(x, t)$ of the Fisher-KPP Equation (10) with $\hat{L}$ of the form (2) that are defined as

$$
\begin{aligned}
& \sigma^{(0)}(t)=\int_{-\infty}^{\infty} u^{(0)}(x, t) d x, \\
& x^{(0)}(t)=\frac{1}{\sigma^{(0)}(t)} \int_{-\infty}^{\infty} x u^{(0)}(x, t) d x, \\
& \alpha^{(k)}(t)=\frac{1}{\sigma^{(0)}(t)} \int_{-\infty}^{\infty} \Delta x^{k} u^{(0)}(x, t) d x .
\end{aligned}
$$

In the class of functions $\mathcal{P}_{t}^{D_{1}}$, we choose

$$
X\left(t, D_{1}\right)=x^{(0)}\left(t, D_{1}\right) .
$$

Then the Fischer-KPP Equation (10), (2), with the coefficients determined by the expansions (23) and (24), can be written as follows:

$$
\begin{aligned}
& -D_{1} u_{t}^{(0)}(x, t)+D_{1}^{2} u_{x x}^{(0)}(x, t)+D_{1} a_{0}(t) u^{(0)}(x, t)+D_{1} a_{x}(t) \Delta x u^{(0)}(x, t)- \\
& -D_{1} \varkappa \sigma^{(0)}(t)\left[b_{0}(t)+\Delta x b_{x}(t) \sigma^{(0)}(t)\right] u^{(0)}(x, t)=O\left(D_{1}^{2}\right) .
\end{aligned}
$$

Here we have used expressions (25)-(28) and estimates (20)-(22). This equation can be called the semiclassically reduced unperturbed Fisher-KPP Equation (10), (2) with the corresponding accuracy.

Integrating Equation (29) with respect to the space variable $x$ over the infinite interval $(-\infty, \infty)$, and taking into account estimates (20)-(22), we can get the following generalized Verhulst evolution equation for the zero moment $\sigma^{(0)}(t)$ accurate to $O\left(\sqrt{D_{1}}\right)$ :

$$
-\dot{\sigma}^{(0)}(t)+a_{0}(t) \sigma^{(0)}(t)-\varkappa b_{0}(t)\left(\sigma^{(0)}(t)\right)^{2}=0,
$$


where $\dot{\sigma}^{(0)}(t)=d \sigma^{(0)}(t) / d t$. Multiplying (29) by $x$ and integrating over $(-\infty, \infty)$, we obtain the following equation for $x^{(0)}(t)$ accurate to $O\left(\sqrt{D_{1}}\right)$ :

$$
\dot{x}^{(0)}(t)=0,
$$

and, similarly, for $\alpha^{(2)}(t)$, accurate to $O\left(D_{1}^{3 / 2}\right)$, we get

$$
\dot{\alpha}^{(2)}(t)=2 D_{1} .
$$

We call Equations (30)-(32) the Einstein-Ehrenfest system for (29), following [23,29].

Applying initial condition (14) to Equations (25)-(27) yields

$$
\begin{aligned}
& \sigma^{(0)}(0)=\int_{-\infty}^{\infty} \varphi(x) d x, \\
& x^{(0)}(0)=X\left(0, D_{1}\right)=\frac{1}{\sigma^{(0)}(0)} \int_{-\infty}^{\infty} x \varphi(x) d x, \\
& \alpha^{(2)}(0)=\frac{1}{\sigma^{(0)}(0)} \int_{-\infty}^{\infty}\left(x-X\left(0, D_{1}\right)\right)^{2} \varphi(x) d x .
\end{aligned}
$$

In particular, for $a(x, t)=a=$ const and $b(x, y)=b(x-y)$ we have that, in Equation (30), $a_{0}(t)=a$ and $b_{0}(t)=b(0)=b=$ const. Then Equation (30) with initial condition (33) is integrated in explicit form as

$$
\sigma^{(0)}(t)=a\left[e^{-a t}\left(\frac{a}{\sigma^{(0)}(0)}-\varkappa b\right)+\varkappa b\right]^{-1}
$$

Next we will find the leading term in the asymptotic expansion of the Cauchy problem solution for the semiclassically reduced Fisher-KPP Equation (29) with initial condition (14) in the class $\mathcal{P}_{t}^{D_{1}}$.

According to [23], we seek a semiclassical solution to Equation (29) as

$$
u^{(0)}(x, t)=u^{(0,0)}(x, t)+\sqrt{D_{1}} u^{(0,1)}(x, t)+O\left(D_{1}\right),
$$

where $u^{(0,0)}(x, t)$ is the leading term of the semiclassical asymptotics, and $u^{(0,1)}(x, t)$ is a first-order correction term in the class $\mathcal{P}_{t}^{D_{1}}$. Note, that from the initial condition (14), we have

$$
u^{(0,0)}(x, 0)=\varphi(x)
$$

Let us substitute expansion (37) and the moment $\sigma^{(0)}(t)$ determined by Equations (30) and (33) into Equation (29) and take into account estimates (20)-(22) and the fact that both $u^{(0,0)}(x, t)$ and $u^{(0,1)}(x, t)$ have the form of (18). Collecting the lower order terms in $\sqrt{D_{1}}$, we get the following equation for $u^{(0,0)}(x, t)$ :

$$
\hat{L} u(x, t)=-u_{t}^{(0,0)}(x, t)+D_{1} u_{x x}^{(0,0)}(x, t)+\left(a_{0}(t)-\varkappa b_{0}(t) \sigma^{(0)}(t)\right) u^{(0,0)}(x, t)=0 .
$$

Note that this equation yields $\dot{S}\left(t, D_{1}\right)=0$ for $S\left(t, D_{1}\right)$ in $(18)$ and $u^{(0,0)}(x, t) \in \mathcal{P}_{t}^{D_{1}}$.

We can call (39) the semiclassically reduced nonlocal Fisher-KPP Equation (10) for the leading term $u^{(0,0)}(x, t)$ of semiclassical asymptotics (37).

Following [23], we present the solution of the Cauchy problem (38) and (39) in terms of the evolution operator of Equation (39) for the initial function $\varphi(x)$ belonging to the class $\mathcal{P}_{0}^{D_{1}}$ defined by (19). 
To write the evolution operator for Equation (39), consider first the linear diffusion equation with the operator (3) for a function $u(x, t)$

$$
\hat{M}(D) u(x, t)=-u_{t}(x, t)+D u_{x x}(x, t)=0 .
$$

The Green function for this equation, which can be taken from a textbook on mathematical physics (e.g., [35]), reads

$$
G(x, y, t, \tau ; D)=\frac{1}{\sqrt{4 \pi D(t-\tau)}} \exp \left(-\frac{(x-y)^{2}}{4 D(t-\tau)}\right)
$$

where $G(x, y, t, t ; D)=\delta(x-y), \delta(x)$ is the Dirac delta-function. Then the solution of the Cauchy problem for Equation (40) with the initial function $u(x, 0)=\phi(x)$ is

$$
u(x, t)=\hat{U}(t, 0 ; D) \phi(x)=\int_{-\infty}^{\infty} G(x, y, t, 0 ; D) \phi(y) d y .
$$

Here $\hat{U}(t, 0 ; D)$ is the evolution operator for Equation (40).

Unlike the linear diffusion Equation (40), the function $w(t)[\varphi]$ in (39),

$$
w(t)[\varphi]=a_{0}(t)-\varkappa b_{0}(t) \sigma^{(0)}(t),
$$

is a functional that depends on the initial function $\varphi(x)$ through the coefficients $a_{0}(t), b_{0}(t)$, and $\sigma^{(0)}(t)$ of Equation (39). Therefore, Equation (39) is nonlinear.

However, we can write the solution of the Cauchy problem for Equation (39) with $u^{(0,0)}(x, 0)=$ $\varphi(x)$ in terms of a nonlinear evolution operator $\hat{U}_{1}\left(t, \tau ; D_{1}, \varphi\right)$ similar to (42) as follows:

$$
u^{(0,0)}(x, t)=\hat{U}_{1}\left(t, 0 ; D_{1}, \varphi\right) \varphi(x)=\exp \left(\int_{0}^{t} w(s)[\varphi] d s\right) \hat{U}\left(t, 0 ; D_{1}\right) \varphi(x),
$$

where $\hat{U}\left(t, 0 ; D_{1}\right)$ is given by (42) with an obvious replacement of $D$ by $D_{1}$.

In conclusion of this subsection, we note that the solution of the Cauchy problem for the linear diffusion Equation (11), (3) with initial condition (15), according to (42) and (41), reads

$$
v^{(0)}(x, t)=\hat{U}\left(t, 0 ; D_{2}\right) \psi(x)=\int_{-\infty}^{\infty} G\left(x, y, t, 0 ; D_{2}\right) \psi(y) d y .
$$

Here the Green function $G\left(x, y, 0 ; D_{2}\right)$ is given by expression of $(41)$, where $D=D_{2}$.

\subsection{The First-Order Correction to the Perturbation Solution of the Non-Local Fisher-KPP Equation}

The first-order correction $u^{(1)}(x, t)$ to the perturbation solution (9) of the generalized non-local Fisher-KPP Equation (4) is determined by solving the Cauchy problem for the inhomogeneous non-local linear Equation (12) with zero initial condition (16). We construct here the solution $u^{(1)}(x, t)$ in the semiclassical approximation in the same class $\mathcal{P}_{t}^{D_{1}}$ of trajectory concentrated functions (18), as in Section 3.1. The functional parameters $X\left(t, D_{1}\right)$ and $S\left(t, D_{1}\right)$ of the class $\mathcal{P}_{t}^{D_{1}}$ are assumed to be found when constructing the leading term $u^{(0,0)}(x, t)$ in (44), and for them we have $\dot{X}\left(t, D_{1}\right)=\dot{S}\left(t, D_{1}\right)=0$.

By analogy with (37), the function $u^{(1)}(x, t)$ can be taken as

$$
u^{(1)}(x, t)=u^{(1,0)}(x, t)+\sqrt{D_{1}} u^{(1,1)}(x, t)+O\left(D_{1}\right),
$$


where $u^{(1,0)}(x, t)$ is the leading-order term, and $u^{(1,1)}(x, t)$ is the first-order correction in the semiclassical asymptotic solution $u^{(1)}(x, t)$ of the problem (12) and (16) in the class $\mathcal{P}_{t}^{D_{1}}$. From the initial condition (16), we have

$$
u^{(1,0)}(x, 0)=0 .
$$

Substituting (46) and expansions (23) and (24) into Equation (12) and doing in the same way as we did to obtain Equation (39) for the function $u^{(0,0)}(x, t)$, we arrive at the following equation for the function $u^{(1,0)}(x, t)$ :

$$
\begin{aligned}
& -u_{t}^{(1,0)}(x, t)+D_{1} u_{x x}^{(1,0)}(x, t)+w(t)[\varphi] u^{(1,0)}(x, t)- \\
& -\varkappa b_{0}(t) \sigma^{(1)}(t) u^{(0,0)}(x, t)-f\left(u^{(0,0)}, v^{(0)}\right)(x, t)=0 .
\end{aligned}
$$

Here we use the following notations: $a_{0}(t)$ and $b_{0}(t)$ are defined in (23), (24); in view of (37) for $\sigma^{(0)}(t)$ given in (25), we have $\sigma^{(0)}(t)=\int_{-\infty}^{\infty} u^{(0,0)}(x, t) d x ; w(t)[\varphi]$ has the form of (43), and $\sigma^{(1)}(t)$ is defined as

$$
\sigma^{(1)}(t)=\int_{-\infty}^{\infty} u^{(1,0)}(x, t) d x
$$

The function $f\left(u^{(0,0)}, v^{(0)}\right)(x, t)$ in Equation (48) can be considered known, as $u^{(0,0)}(x, t)$ and $v^{(0)}(x, t)$ are found in (44) and (45).

Integrating Equation (48) with respect to $x$ over $(-\infty, \infty)$, we arrive at the following equation for the zero moment $\sigma^{(1)}(t)$ :

$$
-\dot{\sigma}^{(1)}(t)+\bar{w}(t)[\varphi] \sigma^{(1)}(t)-z(t)=0,
$$

where the following notation is used:

$$
\begin{aligned}
& \bar{w}(t)[\varphi]=a_{0}(t)-2 \varkappa b_{0}(t) \sigma^{(0)}(t), \\
& z(t)[\varphi]=\int_{-\infty}^{\infty} f\left(u^{(0,0)}, v^{(0)}\right)(x, t) d x .
\end{aligned}
$$

From (47) and (49) we get $\sigma^{(1)}(0)=0$. Integration of the linear ODE (50) with this initial condition yields

$$
\sigma^{(1)}(t)=-\int_{0}^{t} \exp \left(\int_{\tau}^{t} \bar{w}(s)[\varphi] d s\right) z(\tau)[\varphi] d \tau .
$$

In view of (43) and

$$
h(x, t)[\varphi]=\varkappa b_{0}(t) \sigma^{(1)}(t) u^{(0,0)}(x, t)+f\left(u^{(0,0)}, v^{(0)}\right)(x, t),
$$

we can rewrite Equation (48) as

$$
-u_{t}^{(1,0)}(x, t)+D_{1} u_{x x}^{(1,0)}(x, t)+w(t)[\varphi] u^{(1,0)}(x, t)-h(x, t)[\varphi]=0 .
$$

Note that the functions $w(t)[\varphi]$ and $h(x, t)[\varphi]$ in Equation (55) do not depend on $u_{t}^{(1,0)}(x, t)$ and are considered to be known. Therefore, the solution of the Cauchy problem for (55) with initial condition 
(47) can be written in the standard way in terms of the Green function (41) and the Duhamel integral (see, e.g., [35]):

$$
u^{(1,0)}(x, t)=-\int_{0}^{t} \exp \left(\int_{\tau}^{t} w(s)[\varphi] d s\right) \hat{U}\left(t, \tau ; D_{1}\right) h(x, \tau)[\varphi] d \tau
$$

Here, we have

$$
\hat{U}\left(t, \tau ; D_{1}\right) h(x, t)[\varphi]=\int_{-\infty}^{\infty} G\left(x, y, t, \tau ; D_{1}\right) h(y, \tau)[\varphi] d y,
$$

and $G\left(x, y, t, \tau ; D_{1}\right)$ has the form of (41) with $D$ replaced by $D_{1}$.

Finally, similar to (55)-(57), the first-order correction $v^{(1)}(x, t)$ defined by (13) and (17) can be found as

$$
\begin{aligned}
& v^{(1)}(x, t)=-\int_{0}^{t} \hat{U}\left(t, \tau ; D_{2}\right) g\left(u^{(0,0)}, v^{(0)}\right)(x, \tau) d \tau \\
= & -\int_{0}^{t} d \tau \int_{-\infty}^{\infty} G\left(x, y, t, \tau ; D_{2}\right) g\left(u^{(0,0)}, v^{(0)}\right)(y, \tau) d y,
\end{aligned}
$$

where, similar to (56), $G\left(x, y, t, \tau ; D_{2}\right)$ is given by (41) with $D$ replaced by $D_{2}$.

\section{Symmetry Operators}

Although the approximate solutions to the model Equations (4) and (5) were constructed above in terms of evolution operators, the symmetry properties of these equations are also of interest, for instance as an additional way of finding solutions to the equations, more direct in some cases. On the other hand, symmetries are fundamental properties of the laws governing the system states and evolution and, therefore, deserve attention.

As the semiclassically reduced Equation (39) is the key in constructing approximate solutions to the system (4) and (5) under consideration, we will focus on the symmetries of (39).

For any equation, the concept of the symmetry operator is important. By definition, the symmetry operator maps every solution of the equation to a solution.

Group analysis of differential equations [9-12] and some generalizations [13-18] study Lie groups of symmetry operators. The infinitesimal generators of the Lie groups (symmetries of an equation) can be effectively found in many cases, which motivates wide use of symmetries.

The sets of symmetry operators that do not form a Lie group is much harder to find. However, for some special types of equations, this is possible. For example, families of symmetry operators are constructed for the nonlocal generalization of the Gross-Pitaevskii equation in [28], and for the multidimensional nonlocal Fisher-KPP equation in [29] in the framework of the semiclassical approximation method.

Here, we briefly describe the construction of a family of symmetry operators and a set of corresponding solutions for the semiclassically reduced Equation (39) according to [29].

Let us find the general solution of the Einstein-Ehrenfest dynamical system (30) and (31) for the first moments $\sigma^{(0)}(t)$ and $x^{(0)}(t)$ without imposing the initial conditions (33) and (34). Denote the general solution by

$$
\sigma^{(0)}(t)=y(t, \vec{C}), \quad x^{(0)}=C_{2} .
$$

Here $\vec{C}=\left(C_{1}, C_{2}\right)$ are the arbitrary constants of integration.

Application of initial conditions (33) and (34) to the general solution (59) makes $\vec{C}$ a functional dependent on the initial function $\varphi(x), \vec{C}=\vec{C}[\varphi]$. 
For the solution $u^{(0,0)}(x, t)$ of the reduced Equation (39) with (38), $u^{(0,0)}(x, 0)=\varphi(x)$, the following property holds $[23,29]$ :

$$
\vec{C}[\varphi]=\vec{C}\left[u^{(0,0)}\right](t),
$$

where $u^{(0,0)}(x, t)$ is the solution of the reduced Equation (39) with the initial condition (38), $u^{(0,0)}(x, 0)=\varphi(x)$. That is, $\vec{C}\left[u^{(0,0)}\right](t)$ is an integral of Equation (39).

Denote by $w(t, \vec{C})$ a function of the form (43) whose coefficients $a_{0}(t), b_{0}(t)$ and $\sigma^{(0)}(t)$ are obtained from (23), (24) with the use of (59),

$$
w(t, \vec{C})=a_{0}(t, \vec{C})-\varkappa b_{0}(t, \vec{C}) y(t, \vec{C}),
$$

and Equation (30) for the general solution $y(t, \vec{C})$ reads

$$
-\dot{y}(t, \vec{C})+w(t, \vec{C}) y(t, \vec{C})=0 .
$$

Substituting $w(t, \vec{C})$ instead of $w(t)[\varphi]$ defined by (43) into the reduced Equation (39), we obtain a linear equation for a function $\tilde{u}(x, t)$ :

$$
\hat{L}(t, \vec{C}) \tilde{u}(x, t)=\left(-\partial_{t}+D_{1} \partial_{x x}+w(t, \vec{C})\right) \tilde{u}(x, t)=0 .
$$

Denote a solution of Equation (63) by $\tilde{u}(x, t ; \vec{C})$.

We call (63) the associated linear equation (ALE) for the semiclassically reduced Equation (39) according to $[23,29]$. Note that the role of the ALE is played here by the classical diffusion equation with the additional term $w(t, \vec{C})$.

The relationship between the solutions of the Cauchy problem for Equation (39) with initial function (38) and for Equation (63) with the same initial condition, $\tilde{u}(x, 0 ; \vec{C})=\varphi(x)$, is given by the statement [23,29]:

$$
u^{(0,0)}(x, t)=\tilde{u}(x, t ; \vec{C}[\varphi]), \quad \tilde{u}(x, 0 ; \vec{C}[\varphi])=\varphi(x) .
$$

We now proceed to construct the symmetry operators for Equation (39).

Let $u^{(0,0)}(x, t)$ be the solution of (39) with $u^{(0,0)}(x, 0)=\varphi(x)$. If $\hat{A}(t)$ is a symmetry operator for Equation (39), then

$$
u_{A}^{(0,0)}(x, t)=\hat{A}(t) u^{(0,0)}(x, t)
$$

is the solution of Equation (39) with the initial condition

$$
u_{A}^{(0,0)}(x, 0)=\hat{a} \varphi(x)=\varphi_{a}(x),
$$

where $\hat{a}$ is the initial operator,

$$
\hat{A}(x, 0)=\hat{a} .
$$

In what follows we assume $\hat{a}$ to be a linear differential operator.

The solutions $u_{A}^{(0,0)}(x, t)$ and $u^{(0,0)}(x, t)$ (defined in (65)) of the reduced Equation (39) can be presented in terms of the solutions $\tilde{u}(x, t ; \vec{C})$ of the associated linear Equation (63):

$$
u^{(0,0)}(x, t)=\tilde{u}(x, t ; \vec{C}[\varphi]), \quad u_{A}^{(0,0)}(x, t)=\tilde{u}\left(x, t ; \vec{C}\left[\varphi_{a}\right]\right),
$$

where $\varphi_{a}$ is given by (66). 
Consider two sets of arbitrary constants $\vec{C}^{\prime}, \vec{C}$ and the corresponding operators $\hat{L}\left(t, \vec{C}^{\prime}\right)$ and $\hat{L}(t, \vec{C})$ of the associated linear Equation (63).

In view of (64) and (68), we introduce a linear operator $\hat{M}\left(t, \vec{C}^{\prime}, \vec{C}\right)$ intertwining the linear operators $\hat{L}\left(t, \vec{C}^{\prime}\right)$ and $\hat{L}(t, \vec{C})$ :

$$
\hat{L}\left(t, \vec{C}^{\prime}\right) \hat{M}\left(t, \vec{C}^{\prime}, \vec{C}\right)=\hat{R}\left(t, \vec{C}^{\prime}, \vec{C}\right) \hat{L}(t, \vec{C}),
$$

with the condition

$$
\left.\hat{M}\left(t, \vec{C}^{\prime}, \vec{C}\right)\right|_{t=0}=\hat{a}
$$

Here $\hat{R}\left(t, \vec{C}^{\prime}, \vec{C}\right)$ is a Lagrangian multiplier operator.

Then the symmetry operator $\hat{A}(t)$ in $(65)$ can be defined by its action on the function $u^{(0,0)}(x, t)$ in terms of the operators $\hat{M}\left(t, \vec{C}^{\prime}, \vec{C}\right)$ as

$$
\hat{A}(t) u^{(0,0)}(x, t)=\hat{M}\left(t, \vec{C}[\hat{a} \varphi], \vec{C}\left[u^{(0,0)}\right]\right) u^{(0,0)}(x, t) .
$$

Here $\vec{C}[\hat{a} \varphi]$ and $\vec{C}\left[u^{(0,0)}\right]$ are the functionals described above, and (60) is taken into account.

The following expression sets a wide class of linear intertwining operators $\hat{M}\left(t, \vec{C}^{\prime}, \vec{C}\right)$ :

$$
\hat{M}\left(t, \vec{C}^{\prime}, \vec{C}\right)=\hat{D}\left(t, \vec{C}^{\prime}, \vec{C}\right) \hat{B}(t, \vec{C}) .
$$

Here, $\hat{D}\left(t, \vec{C}^{\prime}, \vec{C}\right)$ is a linear intertwining operator defined by

$$
\hat{L}\left(t, \vec{C}^{\prime}\right) \hat{D}\left(t, \vec{C}^{\prime}, \vec{C}\right)=\hat{D}\left(t, \vec{C}^{\prime}, \vec{C}\right) \hat{L}(t, \vec{C}), \quad \hat{D}\left(0, \vec{C}^{\prime}, \vec{C}\right)=\mathbb{I},
$$

where $\mathbb{I}$ is the identity operator. We call $\hat{D}\left(t, \vec{C}^{\prime}, \vec{C}\right)$ the fundamental intertwining operator for $\hat{L}\left(t, \vec{C}^{\prime}\right)$ and $\hat{L}(t, \vec{C})$. With the use of (59), (61)-(63) it is immediately verified that

$$
\hat{D}\left(t, \vec{C}^{\prime}, \vec{C}\right)=\frac{y\left(t, \vec{C}^{\prime}\right) y(0, \vec{C})}{y(t, \vec{C}) y\left(0, \vec{C}^{\prime}\right)} \mathbb{I}
$$

satisfies (73)

By $\hat{B}(t, \vec{C})$ in (72) we denote the symmetry operator of ALE (63), satisfying the conditions

$$
[\hat{L}(t, \vec{C}), \hat{B}(t, \vec{C})]=0, \quad \hat{B}(0, \vec{C})=\hat{a},
$$

where $[\hat{L}, \hat{B}]=\hat{L} \hat{B}-\hat{B} \hat{L}$ is the commutator of $\hat{L}$ and $\hat{B}$.

The Lie algebra of differential symmetry operators for the classical $(1+1)$ diffusion equation is well-known (see, e.g., [36]). In (75) we choose the symmetry operators that commute to zero with the equation operator $\hat{L}(t, \vec{C})$. Then the following first-order linearly independent symmetry operators commuting with $\hat{L}(t, \vec{C})$ can be easily obtained from (75):

$$
\begin{aligned}
& \hat{e}_{1}=\mathbb{I}, \\
& \hat{e}_{2}=\sqrt{2 D_{1}} \partial_{x} \\
& \hat{e}_{3}=\partial_{t}-w(t, \vec{C}), \\
& \hat{e}_{4}=\sqrt{2 D_{1}} t \partial_{x}+\frac{x}{\sqrt{2 D_{1}}},
\end{aligned}
$$

where $\hat{\mathbb{I}}$ is the identity operator. 
Operators (76)-(79) form the basis of the Lie algebra $A_{4,1}$ :

$$
A_{4,1}=\left\{\hat{e}_{1}, \hat{e}_{2}, \hat{e}_{3}, \hat{e}_{4}\right\}
$$

with nonzero commutators

$$
\left[\hat{e}_{2}, \hat{e}_{4}\right]=\hat{e}_{1}, \quad\left[\hat{e}_{3}, \hat{e}_{4}\right]=\hat{e}_{2} .
$$

The four-dimensional nilpotent Lie algebra $A_{4,1}$ arises in the classification of low-dimensional Lie algebras (see, e.g., $[37,38]$ for details).

Let $\hat{h}\left(\hat{e}_{1}, \ldots, \hat{e}_{4}\right)$ denote invariants of $A_{4,1}$, or Casimir elements, also known as Casimir operators. The Casimir operators are elements of the center of the universal enveloping algebra of the Lie algebra $A_{4,1}$ that can be written as [37]

$$
\hat{h}_{1}=\hat{e}_{1}, \quad \hat{h}_{2}=\frac{1}{2} \hat{e}_{2}^{2}-\hat{e}_{1} \hat{e}_{3} .
$$

In view of (76)-(79), Equation (82) yields

$$
\hat{h}_{1}=\hat{\mathbb{I}}, \quad \hat{h}_{2}=\hat{L}(t, \vec{C}),
$$

where $\hat{L}(t, \vec{C})$ is the operator of Equation (63).

Also note that the operators (80), where $w(t, \vec{C})=0$ in (78), are the symmetry operators for the linear diffusion Equation (11) with $\hat{M}\left(D_{2}\right)$ defined by (3). These operators can be used to generate solutions to the diffusion equation.

In the next section we consider an example of solutions of Equation (63) and the functions (45), (56), (58) that give the approximate solution (9) of the model Equations (4) and (5).

\section{Example}

To construct the first-order approximate solution (9),

$$
\begin{aligned}
& u(x, t)=u^{(0,0)}(x, t)+\lambda u^{(1,0)}(x, t)+O\left(\lambda^{2}\right), \\
& v(x, t)=v^{(0)}(x, t)+\lambda v^{(1)}(x, t)+O\left(\lambda^{2}\right),
\end{aligned}
$$

according to (44), (45) and (56), (58), we have to take the function $\varphi(x)$ in (8) from the class $\mathcal{P}_{0}^{D_{1}}$ (see (19)). An example is the Gaussian function

$$
\varphi(x)=A \exp \left(-\frac{\left(x-x_{0}\right)^{2}}{\alpha}\right),
$$

where $A$ is the value of the Gaussian peak, $x_{0}$ is the position of the center of the peak, and $(\alpha / 2)^{1 / 2}$ is the standard deviation where $\alpha / 2$ is the second moment (35).

The initial function $\psi(x)$ in (8) is also taken as

$$
\psi(x)=B \exp \left(-\frac{\left(x-\bar{x}_{0}\right)^{2}}{\beta}\right),
$$

where $B, \bar{x}_{0}$, and $\beta$ have the same meaning as $A, x_{0}$, and $\alpha$ in (85). 
Applying (44) to $\varphi(x)$ in (85) and (45) to $\psi(x)$ in (85) and calculating the convolutions of the Gaussian functions, we get

$$
u^{(0,0)}(x, t)=A e^{\int^{t} w(s)[\varphi] d s}\left(\frac{\alpha}{4 D_{1} t+\alpha}\right)^{\frac{1}{2}} \exp \left(-\frac{\left(x-x_{0}\right)^{2}}{4 D_{1} t+\alpha}\right),
$$

and

$$
v^{(0)}(x, t)=B\left(\frac{\beta}{4 D_{2} t+\beta}\right)^{\frac{1}{2}} \exp \left(-\frac{\left(x-\bar{x}_{0}\right)^{2}}{4 D_{2} t+\beta}\right)
$$

The function $w(s)[\varphi]$ in (43) is given by (30), (33), and (43). For instance, if we take $a_{0}(t)=a=$ const, $b_{0}(t)=b=$ const, and $A=(\pi \alpha)^{-\frac{1}{2}}$ in (85), then (34) yields $\sigma^{(0)}(0)=1$ and from (36) and (43) we have

$$
\begin{aligned}
\sigma^{(0)}(t) & =a e^{a t}\left(a-\varkappa b\left(1-e^{a t}\right)\right)^{-1}, \\
w(t)[\varphi] & =a\left[1-\varkappa b e^{a t}\left(a-\varkappa b\left(1-e^{a t}\right)\right)^{-1}\right],
\end{aligned}
$$

respectively. Integration of $w(t)[\varphi]$ from 0 to $t$ gives

$$
\int_{0}^{t} w(s)[\varphi] d s=a t-\log \left[1-\frac{\varkappa b}{a}\left(1-e^{a t}\right)\right] .
$$

From Equations (54), (56), (57), (87), and (88) for $f(u, v)(x, t)$ and $g(u, v)(x, t)$ of the form (6) and (7), respectively, we come to the following expression for $u^{(1.0)}(x, t)$ :

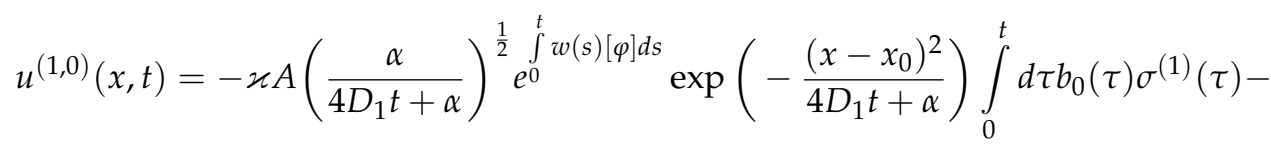

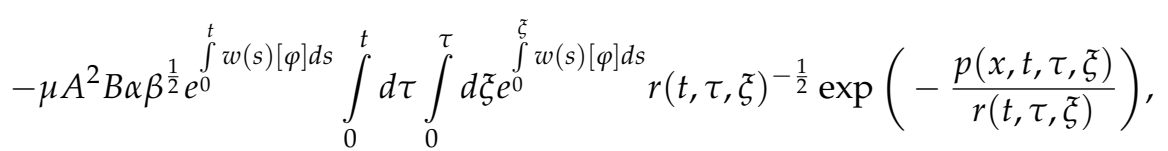

where we use the following designations: $n(\tau)=4 D_{1} \tau+\alpha, k(\xi)=4 D_{2} \xi+\beta$,

$$
\begin{gathered}
r(t, \tau, \xi)=4 D_{1}(t-\tau)(n(\tau) n(\xi)+(n(\tau)+n(\xi)) k(\xi))+n(\tau) n(\xi) k(\xi), \\
p(x, t, \tau, \xi)=\left(x-x_{0}\right)^{2} k(\xi)(n(\tau)+n(\xi))+\left(x-\bar{x}_{0}\right)^{2} n(\tau) n(\xi)+\left(x_{0}-\bar{x}_{0}\right)^{2} 4 D_{1}(t-\tau)(n(\tau)+n(\xi)) .
\end{gathered}
$$

The function $\sigma^{(1)}(t)$ in (89) is given by Equations (52)-(53). Using the function $z(t)[\varphi]$ in (52) for $u^{(0,0)}(x, t), v^{(0)}(x, t)$, and $f\left(u^{(0,0)}, v^{(0)}\right)(x, t)$ of the form (87), (88), and (6), respectively, we have

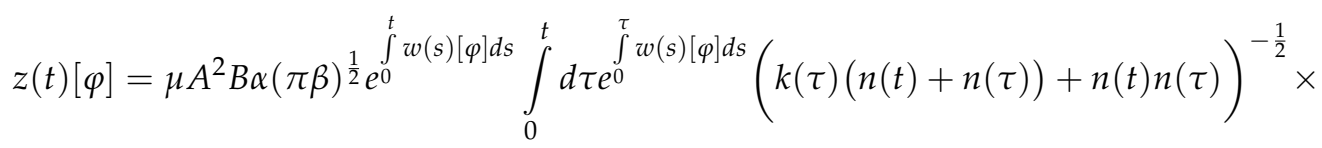

$$
\begin{aligned}
& \times \exp \left\{-\frac{\left(x_{0}-\bar{x}_{0}\right)^{2}(n(t)+n(\tau))}{k(\tau)(n(t)+n(\tau))+n(t) n(\tau)}\right\} .
\end{aligned}
$$


Then the function $\sigma^{(1)}(t)$ given by (53) becomes

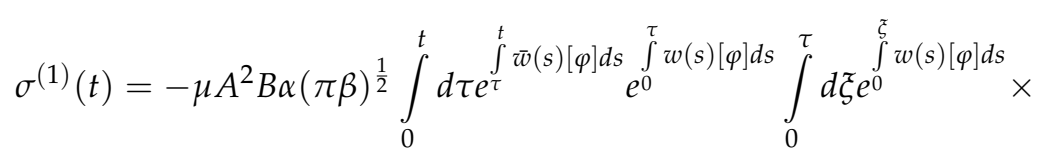

$$
\begin{aligned}
& \times(k(\xi)(n(\tau)+n(\xi))+n(\tau) n(\xi))^{-\frac{1}{2}} \exp \left\{-\frac{\left(x_{0}-\bar{x}_{0}\right)^{2}(n(\tau)+n(\xi))}{k(\xi)(n(\tau)+n(\xi))+n(\tau) n(\xi)}\right\}
\end{aligned}
$$

From (58), (87), and (88) we get

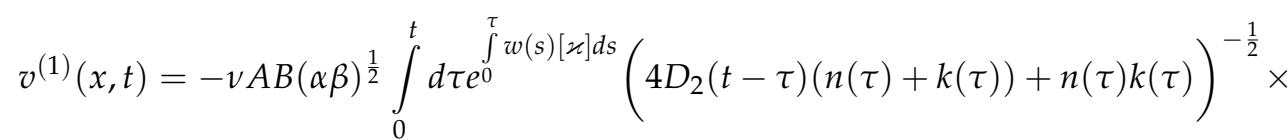

$$
\begin{aligned}
& \times \exp \left\{-\frac{k(\tau)\left(x-x_{0}\right)^{2}+n(\tau)\left(x-\bar{x}_{0}\right)^{2}+4 D_{2}(t-\tau)\left(x_{0}-\bar{x}_{0}\right)^{2}}{4 D_{2}(t-\tau)(n(\tau)+k(\tau))+n(\tau) k(\tau)}\right\} .
\end{aligned}
$$

Expressions (89) and (92) are rather cumbersome. Simpler formulas can be obtained by setting $\bar{x}_{0}=x_{0}$ and passing to the limit $\beta \rightarrow \infty$. The limit means that the initial density (86) is distributed homogeneously over the space, $v(x, 0)=\psi(x) \rightarrow B$. Then Equations (89) and (92) yield

$$
\begin{aligned}
& u^{(1,0)}(x, t)=-\varkappa A\left(\frac{\alpha}{4 D_{1} t+\alpha}\right)^{\frac{1}{2} e^{t} w(s)[\varphi] d s} \exp \left(-\frac{\left(x-x_{0}\right)^{2}}{4 D_{1} t+\alpha}\right) \int_{0}^{t} d \tau b_{0}(\tau) \sigma^{(1)}(\tau)-
\end{aligned}
$$

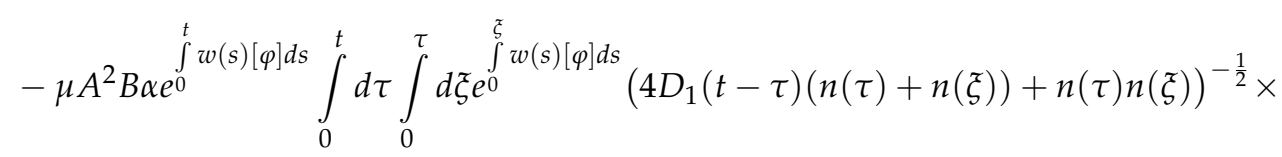

$$
\begin{aligned}
& \times \exp \left\{-\frac{\left(x-x_{0}\right)^{2}(n(\tau)+n(\xi))}{4 D_{1}(t-\tau)(n(\tau)+n(\xi))+n(\tau) n(\xi)}\right\},
\end{aligned}
$$

and

$$
\begin{aligned}
& v^{(1)}(x, t)=-v A B \alpha^{\frac{1}{2}} \int_{0}^{t} d \tau e^{\int_{0}^{\tau} w(s)[\varkappa] d s}\left(4 D_{2}(t-\tau)+4 D_{1} \tau+\alpha\right)^{-\frac{1}{2}} \times \\
& \times \exp \left(-\frac{\left(x-x_{0}\right)^{2}}{4 D_{2}(t-\tau)+4 D_{1} \tau+\alpha}\right) .
\end{aligned}
$$

Equations (90) and (91) take the form

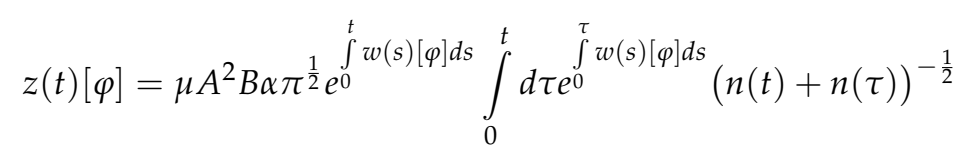

and

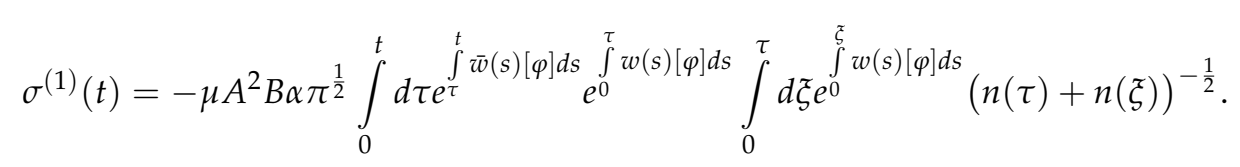

For the pair interaction function

$$
f(u, v)(x, t)=\mu u(x, t) v(x, t)
$$


and $g(u, v)(x, t)$ given by (7), similar to (89), we find

$$
\begin{aligned}
& u^{(1,0)}(x, t)=-\varkappa A\left(\frac{\alpha}{4 D_{1} t+\alpha}\right)^{\frac{1}{2} e^{t} w(s)[\varphi] d s} \exp \left(-\frac{\left(x-x_{0}\right)^{2}}{4 D_{1} t+\alpha}\right) \int_{0}^{t} d \tau b_{0}(\tau) \sigma^{(1)}(\tau)- \\
& -\mu A B(\alpha \beta)^{\frac{1}{2}} e^{t} w(s)[\varphi] d s \int_{0}^{t} d \tau\left(4 D_{1}(t-\tau)(n(\tau)+k(\tau))+n(\tau) k(\tau)\right)^{-\frac{1}{2}} \times \\
& \times \exp \left\{-\frac{k(\tau)\left(x-x_{0}\right)^{2}+n(\tau)\left(x-\bar{x}_{0}\right)^{2}+4 D_{1}(t-\tau)\left(x_{0}-\bar{x}_{0}\right)^{2}}{4 D_{1}(t-\tau)(n(\tau)+k(\tau))+n(\tau) k(\tau)}\right\} .
\end{aligned}
$$

For the functions $z(t)[\varphi]$ and $\sigma^{(1)}(t)$ we have

$$
z(t)[\varphi]=\mu A B(\pi \alpha \beta)^{\frac{1}{2}} e^{\int^{0} w(s)[\varphi] d s}(n(t)+k(t))^{-\frac{1}{2}} \exp \left(-\frac{\left(x_{0}-\bar{x}_{0}\right)^{2}}{n(t)+k(t)}\right)
$$

and

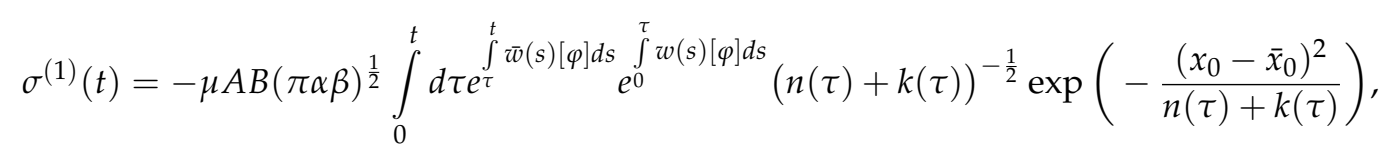

respectively.

For $\bar{x}_{0}=x_{0}$ and $\beta \rightarrow \infty$, we obtain

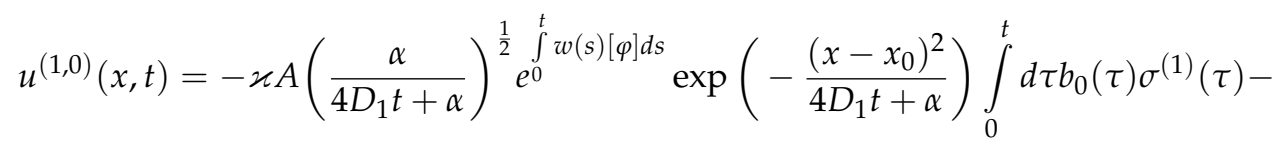

$$
\begin{aligned}
& -\mu A B \alpha^{\frac{1}{2}} t e^{\int^{0} w(s)[\varphi] d s}\left(4 D_{1} t+\alpha\right)^{-\frac{1}{2}} \exp \left(-\frac{\left(x-x_{0}\right)^{2}}{4 D_{1} t+\alpha}\right), \\
& z(t)[\varphi]=\mu A B(\pi \alpha)^{\frac{1}{2}} e^{t} \int^{t} w(s)[\varphi] d s
\end{aligned}
$$

and

$$
\sigma^{(1)}(t)=-\mu A B(\pi \alpha)^{\frac{1}{2}} \int_{0}^{t} d \tau e^{\tau} \bar{t} \bar{w}(s)[\varphi] d s \int_{e^{0}}^{\tau} w(s)[\varphi] d s
$$

The expressions for $u^{(0,0)}(x, t), u^{(1,0)}(x, t), v^{(0)}(x, t)$, and $v^{(1)}(x, t)$ obtained give us some insight into the behavior of the approximate solution (83), (84).

Equation (87) shows that the leading-order term $u^{(0,0)}(x, t)$ of the approximate solution (83) is similar to the initial Gaussian distribution (85) whose peak height and variance evolve with time. The same is true for $v^{(0)}(x, t)$ in (88) and (86).

In the first-order correction $u^{(1,0)}(x, t)$ given by (89), the second summand, caused by the interaction (6), is the time integral (a superposition) of Gaussians whose peak positions and peak values vary with time. This results in a spatial blur of the initial Gaussian (85) in the evolution process that can be meant as the initial stage of pattern formation. This process also affects the density $v^{(1)}(x, t)$ of the form (92). A similar property holds for (96). 
Unlike (89), (96), and (92), expressions (93), (94) and (97) describe the evolution of a Gaussian function with a fixed mean, a time-varying peak value, and a time-varying variance. This process is not considered to be patterning. It may be presumed that the latter will manifest itself in higher approximations.

Finally, it should be noted that the family of solutions (83), (84) in explicit form, if available, can be substantially extended by using the symmetry operators described in Section 4. For example, consider the set of symmetry operators $\left(\hat{a}^{(+)}(t)\right)^{n}$, where $n=0,1, \ldots, \hat{a}^{(+)}(t)=\hat{e}_{2}-\hat{e}_{4}, \hat{e}_{2}$ and $\hat{e}_{4}$ are given in (77)-(79). According to (71), we can generate a set of solutions $u_{n}^{(0,0)}(x, t)=$ $\hat{M}\left(t, \vec{C}\left[\left(\hat{a}^{(+)}(t)\right)^{n} \varphi\right], \vec{C}\left[u^{(0,0)}\right]\right) u^{(0,0)}(x, t)$ starting from $u^{(0,0)}(x, t)$ of the form $(87)$. Such solutions were constructed in explicit form in [29]. Then, using the operators (56) and (58), we can construct the approximate solutions (83), (84).

\section{Conclusions}

A two-parameter approximation is proposed for a $(1+1)$-dimensional two-component non-local RD population model which describes a population interacting with an active substance.

The small parameters used are the population diffusion coefficient and the parameter of interaction between the population and the substance.

First, we apply the first-order perturbation method to the model equations in the interaction parameter and obtain that finding the leading term of the perturbation solution is reduced to solving the non-local generalized Fisher-KPP equation.

Next, we construct an approximate solution to this equation using the WKB-Maslov method of semiclassical asymptotics with the diffusion coefficient as the asymptotic parameter.

As the dynamics of the active substance is described by the diffusion equation, its solution does not cause additional difficulties. To extend the applicability of the approach, we describe a family of symmetry operators for the semiclassically reduced Fisher-KPP equation. Finally, we illustrate the general formulas by an example taking the Gaussian functions as the initial conditions for the model equations.

The model describing the combined evolution of a microbial population interacting with an active substance discussed in this work is of obvious interest as it allows one to explore the dynamics of a microbial population and, importantly, the growth of a cell population under the control of external factors.

In particular, different points of view are expressed in the literature, regarding the hypothesis of special properties of strongly diluted solutions of active materials and their influence on biological objects (see, e.g., [25-27]). In this context, a conjecture can be made that such solutions of an active substance possesses some special properties, for instance, fractal properties. In this case, a modification of the model equations can be such that it will take fractal properties into account, for example, with the help of the method of fractional analysis (see, e.g., [39]).

Author Contributions: Conceptualization, A.V.S. and A.Y.T.; methodology, A.V.S.; formal analysis, A.V.S. and A.Y.T.; investigation, A.V.S. and A.Y.T.; validation, A.V.S. and A.Y.T.; writing-original draft, A.V.S.; writing-review \& editing, A.V.S.

Acknowledgments: This work was supported by the Physical Aspects of Release Activity project, Tomsk State Pedagogical University; by Tomsk State University under the International Competitiveness Improvement Program, and by Tomsk Polytechnic University under the International Competitiveness Improvement Program.

Funding: This research received no external funding.

Conflicts of Interest: The authors declare no conflict of interest.

\section{References}

1. Murray, J.D. Mathematical Biology. I. An Introduction, 3rd ed.; Springer: New York, NY, USA, 2001; p. XXIII, 553.

2. Fisher, R.A. The wave of advance of advantageous genes. Annu. Eugen. 1937, 7, 255-369. [CrossRef] 
3. Kolmogorov, A.N.; Petrovskii, I.G.; Piskounov, N.S. A study of the diffusion equation with increase in the amount of substance and its application to a biology problem. Bull. Univ. Moscow Ser. Int. A 1937, 1, 1-16.

4. Fuentes, M.A.; Kuperman, M.N.; Kenkre, V.M. Nonlocal interaction effects on pattern formation in population dynamics. Phys. Rev. Lett. 2003, 91, 158104. [CrossRef] [PubMed]

5. d'Onofrio, A.; Gandolfi, A. Mathematical Oncology 2013. Modeling and Simulation in Science, Engineering and Technology; Springer: New York, NY, USA, 2014; p. 334.

6. Levchenko, E.A.; Shapovalov, A.V.; Trifonov, A.Y. Pattern formation in terms of semiclassically limited distribution on lower dimensional manifolds for the nonlocal Fisher-Kolmogorov-Petrovskii-Piskunov equation. J. Phys. A Math. Theor. 2014, 47. [CrossRef]

7. Levchenko, E.A.; Shapovalov, A.V.; Trifonov, A.Y. Asymptotics semiclassically concentrated on curves for the nonlocal Fisher-Kolmogorov-Petrovskii-Piskunov equation. J. Phys. A Math. Theor. 2016, 49. [CrossRef]

8. Shapovalov, A.V.; Obukhov, V.V. Some aspects of nonlinearity and self-organization in biosystems on examples of localized excitations in the DNA molecule and generalized Fisher-KPP model. Symmetry 2018, 10, 53. [CrossRef]

9. Bluman, G.W.; Cole, J.D. Similarity Methods for Differential Equations; Series Title: Applied Mathematical Sciences, V. 13; Springer: New York, NY, USA, 1974; p. IX, 333.

10. Ovsyannikov, L.V. Group Analysis of Differential Equations; Academic Press: New York, USA, 1982; p. 432.

11. Ibragimov, N.H. Transformation Groups Applied to Mathematical Physics; Mathematics and Its Applications; Soviet Series; D. Reidel Publishing: Dordrecht, The Netherlands, 1985; p. XV, 394.

12. Olver, P.J. Applications of Lie Groups to Differential Equations; Series Title: Graduate Texts in Mathematics, V. 107; Springer: New York, NY, USA, 1993; p. XXVIII, 513.

13. Akhatov, I.S.; Gazizov, R.K.; Ibragimov, N.H. Nonlocal symmetries: a heuristic approach. J. Sov. Math. 1991, 55, 1401-1450. [CrossRef]

14. Chetverikov, V.N.; Kudryavtsev, A.G. Modeling integro-differential equations and a method for computing their symmetries and conservation laws. Am. Math. Soc. Transl. 1995, 167, 1-22.

15. Zawistowski, Z.J. Symmetries of integro-differential equations. Proc. Inst. Mat. NAS Ukraine 2002, 43, $263-270$. [CrossRef]

16. Meleshko, S.V. Methods for Constructing Exact Solutions of Partial Differential Equations: Mathematical and Analytical Techniques with Applications to Engineering; Springer: New York, NY, USA, 2005.

17. Fushchych W.; Shtelen W. On nonlocal transformations. Lett. Nuovo Cim. 1985, 44, 40-42. [CrossRef]

18. Levchenko, E.A.; Shapovalov, A.V.; Trifonov, A.Y. Symmetries of the Fisher-Kolmogorov-Petrovskii-Piskunov equation with a nonlocal nonlinearity in a semiclassical approximation. J. Math. Anal. Appl. 2012, 395, 716-726. [CrossRef]

19. Maslov, V.P. Operational Methods; MIR Pub.: Moscow, Russia, 1976; p. 559.

20. Maslov, V.P. The Complex WKB Method for Nonlinear Equations. I. Linear Theory; Series: Progress in Mathematical Physics (Book 16); Birkhäuser: Basel, Switzerland, 1994; p. 304.

21. Belov, V.V.; Dobrokhotov, S.Y. Semiclassical Maslov asymptotics with complex phases. I. General appoach. Teor. Mat. Fiz. 1992, 130, 215-254;

22. Trifonov, A.Y.; Shapovalov, A.V. The one-dimensional Fisher-Kolmogorov equation with a nonlocal nonlinearity in a semiclassical approximetion. Russ. Phys. J. 2009, 52, 899-911. [CrossRef]

23. Shapovalov, A.V.; Trifonov, A.Y. An application of the Maslov complex germ method to the one-dimensional nonlocal Fisher-KPP equation. Int. J. Geom. Methods Mod. Phys. 2018, 15, 1850102. [CrossRef]

24. Shapovalov, A.V.; Obukhov, V.V. Influence of the environment on pattern formation in the one-dimensional nonlocal Fisher-Kolmogorov-Petrovskii-Piskunov model. Russ. Phys. J. 2018, 6, 1093-1099. [CrossRef]

25. Tarasov, S.A.; Zarubaev, V.V.; Gorbunov, E.A.; Sergeeva, S.A.; Epstein, O.I. Activity of ultra-low doses of antibodies to gamma-interferon against lethal influenza A (H1N1) 2009 virus infection in mice. Antivir. Res. 2012, 93, 219-224. [CrossRef] [PubMed]

26. Nicoll, J.; Gorbunov, E.A.; Tarasov, S.A.; Epstein, O.I. Subetta treatment increases adiponectin secretion by mature human adipocytes in vitro. Int. J. Endocrinol. 2013, 2013, 925874. [CrossRef]

27. Epstein, O. The spatial homeostasis hypothesis. Symmetry 2018, 10, 103. [CrossRef]

28. Lisok, A.L.; Shapovalov, A.V.; Trifonov, A.Y. Symmetry and Intertwining Operators for the Nonlocal Gross-Pitaevskii Equation. Symmetry Integr. Geom. Methods Appl. 2013, 9, 1-21. [CrossRef] 
29. Levchenko, E.A.; Trifonov, A.Y.; Shapovalov, A.V. Symmetry operators of the nonlocal Fisher-KolmogorovPetrovskii-Piskunov equation with a quadratic operator. Russ. Phys. J. 2014, 56, 1415-1426. [CrossRef]

30. Haas, F.; Eliasson, B. Time-dependent variational approach for Bose-Einstein condensates with nonlocal interaction. J. Phys. B At. Mol. Opt. Phys. 2018, 51, 175302. [CrossRef]

31. Bamba, K.; Capozziello, S.; Nojiri, S.; Odintsov, S.D. Dark energy cosmology: The equivalent description via different theoretical models and cosmography tests. Astrophys. Space Sci. 2012, 342, 155-228. [CrossRef]

32. Simbawa, E. Mechanistic model for cancer growth and response to chemotherapy. Comput. Math. Methods Med. 2017, 2017. [CrossRef]

33. Bagrov, V.G.; Belov, V.V.; Trifonov, A.Y. Semiclassical trajectory-coherent approximation in quantum mechanics: I. High order corrections to multidimensional time-dependent equations of Schrödinger type. Ann. Phys. 1996, 246, 231-280. [CrossRef]

34. Belov, V.V.; Trifonov, A.Y.; Shapovalov, A.V. The trajectory-coherent approximation and the system of moments for the Hartree type equation. Int. J. Math. Math. Sci. 2002, 32, 325-370. [CrossRef]

35. Tikhonov, A.N.; Samarskii, A.A. Equations of Mathematical Physics; Pergamon Press: Oxford, UK, $1963 ;$ p. 765.

36. Miller, W. Symmetry and Separation of Variables; Cambridge University Press: Cambridge, UK, 1984; p. 318.

37. Patera, J.; Sharp, R.T.; Winternitz, P.; Zassenhaus, H. Invariants of real low dimension Lie algebras. J. Math. Phys. 1976, 6, 986-994. [CrossRef]

38. Konyaev, A.Y. Classification of Lie algebras with generic orbits of dimension 2 in the coadjoint representation. Sb. Math. 2014, 205, 45-62. [CrossRef]

39. Samko, S.G.; Kilbas, A.A.; Marichev, O.I. Fractional Integrals and Derivatives: Theory and Applications; Gordon and Breach Science Publishers: Langhorne, PA, USA, 1993; p. xxxvi, 976.

(C) 2019 by the authors. Licensee MDPI, Basel, Switzerland. This article is an open access article distributed under the terms and conditions of the Creative Commons Attribution (CC BY) license (http:/ / creativecommons.org/licenses/by/4.0/). 